\title{
Le raccordement métrologique des thermocycleurs au Système International (SI) dans le cadre de l'accréditation ISO 15189
}

\author{
Philippe LE TOUZÉ 1,a \\ ${ }^{I} A+$ METROLOGIE, 191 rue de Vaugirard, 75015 Paris, France
}

\begin{abstract}
Biotechnologies are in full boom among them the molecular biology. Polymerase Chain Reaction (PCR) is the main technology that permits such development. This recent analytical methods allows from a small piece of DNA to obtain a large number of copies (millions) of this very same fragment. PCR is performed with a device named a thermal cycler or a thermocycler. This high-tech equipment realizes 30 to 40 very short temperature run so after 30 minutes DNA is duplicated in a necessary and sufficient quantity. The user distributes with a micropipette his sample in one of the well of the block reaction and push a button: looks like easy! That's how such device is democratized as a routine diagnostic tool in medical laboratories. In order to confirm their competence, medical laboratories must be accredited by the ISO 15189 standard. Laboratory equipment such thermocycler, regarding the critical to the diagnostic provided, must be calibrated by an accredited laboratory (traceability). Without an ISO standard dedicated to perform thermocycler metrology, an internal protocol was created. This protocol is accredited Cofrac and rely to a physical method. This method allows to highlight the fact that thermal profile of thermocycler is unique! In order to compare devices together, to develop a strategy for test routines, to follow the drift of devices, the only solution is a unique and identical calibration program. This methodology should be standardized and give birth to a dedicated ISO. This is one of the next steps. Ensure the traceability of the real time PCR is the new challenge for the accredited metrological companies, by introducing optical metrology inside medical laboratories.
\end{abstract}

\section{Introduction}

La métrologie a fait une entrée remarquée ces dernières années dans le secteur de la biologie médicale, lié au fait de la réforme [1] de ce secteur d'activité.

Cette réforme est issue des constats suivants :

- La biologie médicale est devenue un élément central du parcours de soins des patients, déterminant pour l'élaboration d'environ $60 \%$ des diagnostics.

- La nécessité d'une traçabilité de la qualité des examens de biologie médicale réalisés.

Cette réforme est également l'aboutissement d'études réalisées depuis le milieu des années 2000 : le rapport de l'Inspection Générale des Affaires Sociales (IGAS) en 2006 qui, le premier, a souligné la nécessité de réformer la biologie médicale en constatant que malgré un niveau global de qualité des examens satisfaisant, il restait des insuffisances incompatibles avec les besoins en matière de santé publique. Le rapport dit «Ballereau » de 2008 a jeté les bases de la réforme actuelle.
Cette réforme intervient 33 ans après la dernière réforme générale de la biologie (la loi du 11 juillet 1975) et 20 ans après l'instauration des Sociétés d'Exercice Libéral.

Cette réforme comprend 2 mesures-phares :

- La médicalisation : il s'agit de réaffirmer le rôle du biologiste médical au sein du parcours de soins. Cette reconnaissance se traduit par la création d'une responsabilité pour le biologiste médical qui interprète à présent systématiquement l'ensemble des résultats des examens qu'il réalise.

- L'accréditation : La procédure d'accréditation obligatoire garantit une qualité tracée et prouvée des examens de biologie médicale par une vérification de la compétence d'un laboratoire de biologie médicale (LBM) par ses pairs que sont les autres biologistes médicaux en exercice.

Cette accréditation porte non seulement sur la phase analytique mais également sur les phases pré-analytique (prélèvement et transports jusqu'au lieu de l'analyse) et post analytique (validation du résultat, interprétation biologique).

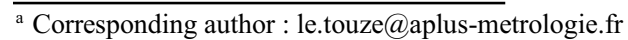


L'accréditation est délivrée par un organisme national d'accréditation sur demande du laboratoire de biologie médicale (LBM). En France, cet organisme est le Comité français d'accréditation (Cofrac) [2]. En Europe, chaque pays n'a qu'un seul organisme d'accréditation.

Cette accréditation est une reconnaissance des compétences techniques et du système de management de la qualité du LBM.

L'accréditation s'obtient en se conférant à l'ISO 15189 [3]. Cette norme internationale spécifie les exigences de qualité et de compétences applicables aux LBM. Cette norme a été révisée en 2012 - troisième édition -, la version précédente datant de 2007.

\section{Un appareil de haute technologie}

Depuis l'invention de la PCR, pléthore de marque et de modèles de thermocycleurs ont été mis sur le marché. La nécessité de fabriquer des thermocyleurs de plus en plus rapide, permettant d'optimiser le process client, a permis à la technologie Peltier de s'imposer majoritairement dans la conception de ceux-ci.

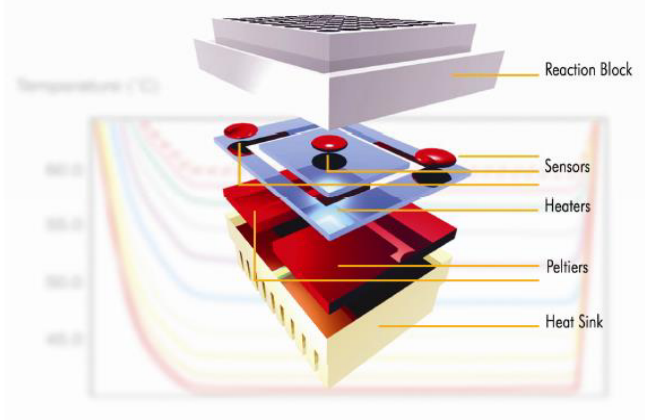

Figure 1. Vue en «sandwich » d'un thermocycleur à effet Peltier [4]

Les dernières avancées en terme de technologie permettent désormais de réaliser une PCR (polymerase chain reaction ou amplification génique [5]) en moins de 30 minutes.

Cette technique d'analyse permet donc de mettre en évidence in vitro des quantités infinitésimales d'A.D.N. par duplications successives exceptionnelles d'un gène en un point d'un chromosome.

\section{Un appareil critique}

Le guide technique d'accréditation SH GTA 01 rév 01 [6] " guide technique d'accréditation en biologie médicale » mentionne qu'il convient au laboratoire de lister ces équipements et matériels ayant une influence sur la qualité du résultat (équipements dit «critiques »).Les équipements critiques demandent un raccordement métrologique et un suivi.

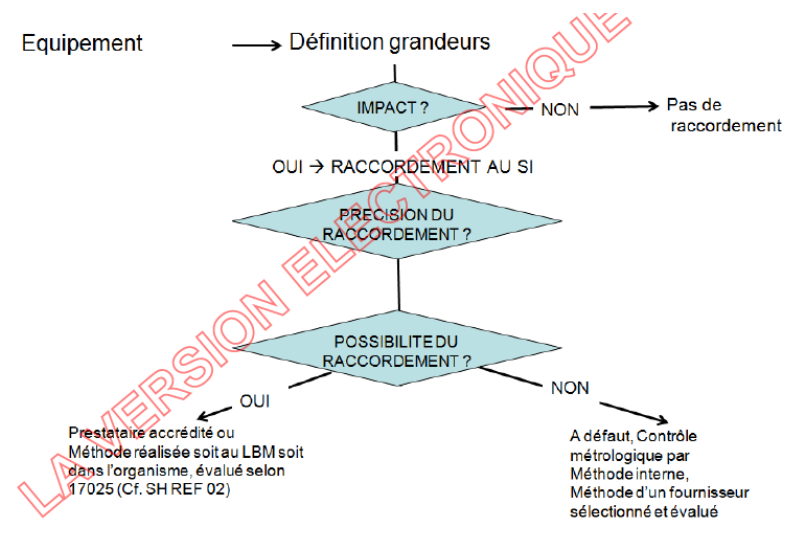

Figure 2. Utilité et pertinence du raccordement, extrait $\mathrm{SH}$ GTA-01 [6]

La dernière version du guide (SH-GTA-01 rev 01 du 15 avril 2015) consacre une page d'annexe (G) au thermocycleur alors que la version précédente n'y consacrait que quelques lignes.

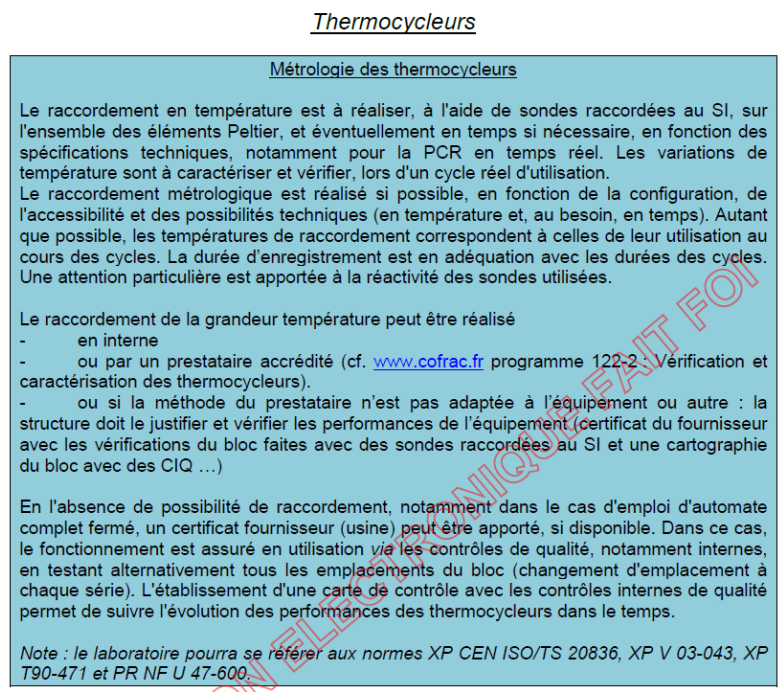

Figure 3. Métrologie des thermocycleurs, extrait SH-GTA-01 [6]

\section{Un haut niveau d'expertise requis}

La métrologie des thermocycleurs porte sur la grandeur température, par méthode physique.

Il faut ainsi pouvoir mesurer de manière fiable des variations de températures extrêmement rapides, sur des temps très courts.

Un puissant système d'acquisition (acquisition de l'ordre de la centaine de millisecondes (ms)), à l'aide de capteurs miniaturisés (unité de volume au sein du bloc de réaction de l'ordre du $\mu \mathrm{l})$, avec une incertitude de mesure de l'ordre du dixième de degré Celsius $\left({ }^{\circ} \mathrm{C}\right)$ doit être mis en œuvre afin de pouvoir raccorder métrologiquement un thermocycleur.

Le prestataire, dans le cadre d'un raccordement par un service support externe, doit être accrédité par le Cofrac. Il est à noter qu'avant 2014, aucune société de métrologie n'était accréditée en France, compliquant de facto la possibilité de raccordement au Système International [7]. 


\section{Absence de référentiel normatif ISO}

Il n'existe pas de norme ISO dédiée à la métrologie d'un thermocycleur.

Le guide SH-GTA-01 [6] mentionne 4 normes différentes !

Certaines n'abordent pas directement la métrologie du thermocycleur en tant que tel, mais plutôt son utilisation dans le périmètre du domaine abordé.

Lorsqu'elles l'abordent, l'approche n'est pas correcte en ce sens où celles-ci visent plus une validation du process client que du thermocycleur en lui-même. De plus, les méthodes proposées diffèrent entre elles.

Cela crée plus de confusion pour l'utilisateur et complique la revue de contrat avec le prestataire accrédité.

Pourtant, l'appareil utilisé pour l'ensemble de ces domaines d'activité reste le même à savoir un thermocycleur.

Les organismes accrédités Cofrac proposent donc une méthode interne.

Il apparait nécessaire dans un avenir proche de mettre en place une norme ISO dédiée à la métrologie du thermocycleur, afin de combler ce manque et d'uniformiser les pratiques. Une méthode normalisée permettra à tous plus d'aisance et de clarté.

\section{Une empreinte thermique unique}

Chaque thermocycleur est unique et se définit par son empreinte thermique.

Les caractéristiques de son empreinte thermique sont :

- Vitesse de montée (ramp phase) en ${ }^{\circ} \mathrm{C} / \mathrm{s}$

- Vitesse de descente (ramp phase) en ${ }^{\circ} \mathrm{C} / \mathrm{s}$

- Overshoot en ${ }^{\circ} \mathrm{C}$

- Undershoot en ${ }^{\circ} \mathrm{C}$

- Temps de maintien (hold time) en $\mathrm{s}$

- Exactitude (accuracy) en ${ }^{\circ} \mathrm{C}$

- Uniformité (uniformity) en ${ }^{\circ} \mathrm{C}$

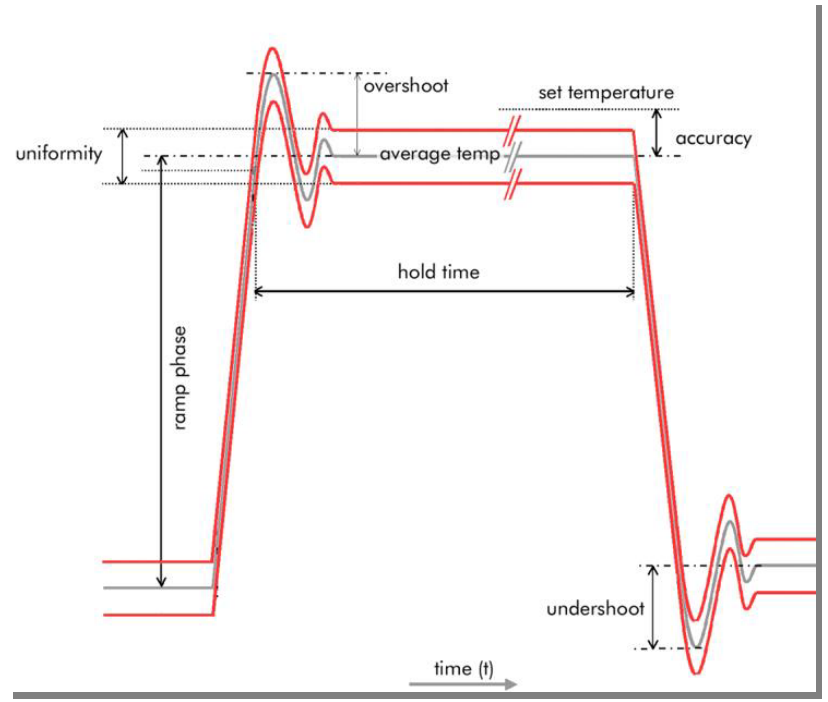

Figure 4. Définition graphique des paramètres caractéristiques

Notre expérience, en tant que société prestataire de métrologie en thermocycleur, nous permet de constater que des différences en termes d'empreinte thermique existe, non pas uniquement entre marques, mais également entre même modèle !

\section{La solution : un programme unique et identique pour tous}

Comme mentionné dans le paragraphe 2, l'offre proposée sur le marché en termes d'achat de thermocycleurs est très variée.

Le parc de thermocycleurs est ainsi hétérogène dans chaque laboratoire.Il est fréquent de constater moins de 2 thermocycleurs identiques au sein d'un même parc d'appareils.

Le nombre d'effets Peltier situés sous le bloc de réaction varie d'une marque à l'autre, d'un modèle à l'autre.

Les algorithmes de calcul des effets Peltier différent également, et cela pour un même appareil.

Comment l'utilisateur va-t-il pouvoir mener une stratégie de choix relative à la gestion de son parc de thermocycleurs?

En d'autres termes, comment peut-il affirmer que tel thermocycleur fonctionne aussi bien que son voisin?

La seule solution réside dans le déroulement d'un programme unique pour l'ensemble du parc.

Cela permet également une analyse dans le temps du comportement de son parc d'instruments (dérive, vieillissement).

Grace à ce programme unique, cela permet également un moyen de comparaison entre services, entre hôpitaux, entre pays, dans le monde entier.

Rappelons que l'un des buts de la réforme de la biologie médicale est de rendre uniforme les pratiques au niveau européen.

\section{Où placer les Contrôles Interne de Qualité (CIQ)?}

Le laboratoire, dans le cadre de la vérification continue des performances lors de l'utilisation du thermocycleur doit assurer un contrôle de qualité interne. Ce contrôle de routine constitue un apport de preuve de la maitrise en continu du processus analytique. Celui-ci est réalisé à l'aide de réactifs, par méthode biologique. La plaque d'essai situé sur le bloc de réaction peut varier de 24 à 1536 puits!

Comme chaque thermocycleur possède une empreinte thermique unique, où placer ces contrôles interne de qualité ? 


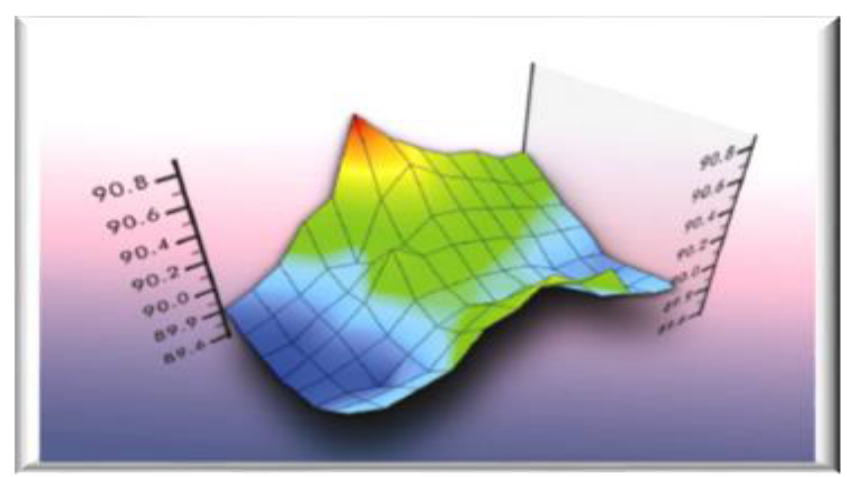

Figure 5. Visualisation de l'uniformité d'un bloc de réaction [4]

Suite à la cartographie par méthode physique, des positions types sur la plaque d'essai peuvent être identifiées, rendant possible une stratégie de choix pour le laboratoire dans le déroulement des contrôles interne de qualité.

\section{La PCR en temps réel - vision d'avenir}

La technique d'amplification génique est en constante évolution. Certains thermocycleurs permettent ainsi une quantification de l'ADN par fluorescence.

Cette mesure, partie intégrante du diagnostic rendu, par cohérence, requiert un raccordement en métrologie et un suivi.

Gageons que la prochaine évolution en termes de prestation de services soit la suivante : permettre au laboratoire d'obtenir une incertitude de mesure sur la valeur de fluorescence exprimée, par méthode physique. La métrologie optique fait son entrée dans le raccordement métrologique des thermocyleurs.

\section{Conclusion}

La réforme de la biologie médicale a rendu obligatoire l'introduction d'une métrologie accréditée au sein des LBM. L'absence de référentiel normatif international dédié à la métrologie thermique du thermocycleur pose problème car on peut constater des pratiques hétérogènes. Le haut niveau d'expertise pratiqué par les prestataires accrédités permet de faire face et d'apporter des solutions pour la réalisation de la métrologie par méthode physique de ce type d'appareil de haute technologie.

$\mathrm{Du}$ fait de son empreinte thermique unique, la seule solution permettant une stratégie de choix dans la gestion d'un parc hétérogène est un programme unique et identique pour tous. Cette méthode permet également une stratégie de choix concernant les contrôles internes de qualité.

Les prestataires accrédités ont su faire acte de réactivité afin de répondre au besoin de raccordement au SI des LBM en terme de PCR dite "classique ».Un nouveau challenge s'offre à eux afin de répondre au besoin de raccordement au SI en terme de PCR en temps réel, en faisant appel aux ressources de la métrologie optique.

\section{Références}

[1]

http://www.sante.gouv.fr/biologie-medicale.html

[2]

http://www.cofrac.fr

[3]

http://www.iso.org/iso/fr/catalogue detail?csnumber=561 15

[4]

Avec l'aimable authorisation de la société cyclertest www.cyclertest.com

[5]

http://www.larousse.fr/dictionnaires/francais/amplificatio $\mathrm{n} / 3110 /$ locution

[6]

http://www.cofrac.fr/documentation/SH-GTA-01

[7] http://www.bipm.org/utils/common/pdf/si_brochure 8 fr .pdf 